Supplement of Geosci. Model Dev., 13, 2925-2944, 2020

https://doi.org/10.5194/gmd-13-2925-2020-supplement

C Author(s) 2020. This work is distributed under

the Creative Commons Attribution 4.0 License.

(c) (1)

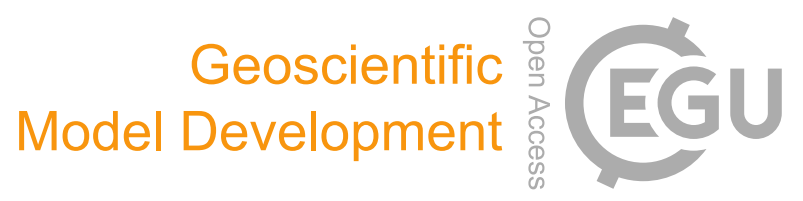

Supplement of

\title{
A multiphase CMAQ version 5.0 adjoint
}

Shunliu Zhao et al.

Correspondence to: Amir Hakami (amir.hakami@ carleton.ca)

The copyright of individual parts of the supplement might differ from the CC BY 4.0 License. 


\title{
CMAQ v5.0 Adjoint User's Manual
}

\author{
The Adjoint Development Team
}

May 22, 2020

\section{Contents}

1 Introduction 2

2 Installation $\quad 2$

2.1 Prerequisites . . . . . . . . . . . . . . . . . . 2

2.2 Retrieve the adjoint . . . . . . . . . . . . . . . . . 2

2.3 Build the builder . . . . . . . . . . . . . . . . . . 3

2.4 Build CMAQ libraries . . . . . . . . . . . . . . . . . . . . . 3

2.5 Build the forward model . . . . . . . . . . . . . . . . . 3

2.6 Build backward sweep executable . . . . . . . . . . . . . . . 4

3 Benchmark the adjoint $\quad 4$

3.1 Run the forward model . . . . . . . . . . . . . . . . . . . . 4

3.2 Create adjoint forcing . . . . . . . . . . . . . . . . . 5

3.3 Run the backward model . . . . . . . . . . . . . . . . . . 5

4 Finite difference test 5

4.1 The finite difference module . . . . . . . . . . . . . 5

4.2 Compare with the adjoint . . . . . . . . . . . . . 6

5 Strategies to implement the adjoint $\quad 6$

5.1 About checkpointing . . . . . . . . . . . . . . 6

5.2 About forcing . . . . . . . . . . . . . . . . . . . . . . . . . . . . . . . . . . .

5.3 About adjoint sensitivities . . . . . . . . . . . . . . . 9

$\begin{array}{ll}\text { References } & 10\end{array}$ 


\section{Introduction}

The CMAQ adjoint is an active open-source development project of academic collaborators that augments CMAQ model development by the U.S. EPA to estimate sensitivities of functions of modeled concentrations with respect to initial concentrations or emissions parameters.

The adjoint model is comprised of three major components:

- the forward model, which generates checkpointing files for the science processes and average concentrations needed for forcing calculation

- the forcing generator, which creates forcing files

- the backward model, which reads the checkpointing and forcing files to calculate the sensitivity parameters.

To run the adjoint model, you need to

- prepare your system with the required libraries

- compile the adjoint source code with your favorite compilers

- run sequentially the forward model, the forcing generator, and the backward model.

This document is to provide a general guidance on building and running the adjoint model. For a detailed description of the adjoint, please refer to [Shunliu Zhao, 2019].

The latest version of this document comes with the model.

\section{Installation}

\subsection{Prerequisites}

To retrieve, compile, and run the adjoint model, the following software are required.

- Git

- netCDF 3.6.3 or later

- I/O API 3.0 or later

- MPI, e.g. OpenMPI. MPICH2, or MVAPICH2

- PGI/Intel/GCC Fortran and C compilers

\subsection{Retrieve the adjoint}

A copy of the CMAQ adjoint code can be obtained using Git.

git clone ssh://git@adjoint.colorado.edu/yanko.davila/cmaq_adj.git

To create a local Git branch for testing, change into the model directory and execute the following command.

git checkout -b test origin/5.0 


\subsection{Build the builder}

The adjoint uses the CMAQ model builder to assemble and/or compile the code ([CMASWIKI, 2015]);

To build the builder from source, change directory to \$ADJHOME/BLDMAKE_git, customize the Makefile if necessary, and then make:

cd \$ADJHOME/BLDMAKE_git

make I\& tee make.bld.log

Here, \$ADJHOME is your work directory for the adjoint.

Ensure that bldmake is created.

\subsection{Build CMAQ libraries}

The STENEX and PARIO libraries from CMAQ are required for the adjoint for parallel job management and parallel input/output. Serial jobs needs only the NOOP version of STENEX and not PARIO. More details about the two libraries could be found at [CMASWIKI, 2015].

To compile STENEX, change directory to the corresponding directory, revise the Makefile, and then make:

cd \$ADJHOME/stenex/se

make I\& tee make.stenex.se.log

Check the log file for errors and the target directory for libse_snl.a and module files (.mod).

To compile pario,

cd \$ADJHOME/pario

\#\#Revise the Makefile

make I\& tee make.pario.log

Check the log for errors and the target directory for libpario.a and module files (.mod).

\subsection{Build the forward model}

Change directory to scripts/

cd \$ADJHOME/scripts

Using the build script as a template, cmaq_adj/scripts/bldit.adjoint.fwd.sample, create an executable for the forward sweep (comparable to CMAQ).

To configure a specific build, copy bldit.adjoint.fwd.sample to a file specific to your system and then customize the new file.

Execute the script (as exemplified with the sample script below).

./bldit.adjoint.fwd.[system-specific-name] I \& tee bldit.fwd.log

A new directory should be made in \$ADJHOME named BLD_fwd_bnmk. Change to it.

cd ../BLD_fwd_bnmk

Edit the Makefile to ensure compatibility with your system. Then, make the forward sweep executable.

make I\& tee make.fwd.log

Check that the executable ADJOINT_FWD is created. 


\subsection{Build backward sweep executable}

Follow the same procedure for the backward sweep of the model. To configure a specific build, copy bldit.adjoint.bwd.sample to a file specific to your system and then customize the new file.

Execute the script (as exemplified with the sample script below).

./bldit.adjoint.bwd. [system-specific-name] I\& tee bldit.bwd.log

A new directory should be made in \$ADJHOME named BLD_bwd_bnmk. Change to it.

cd .../BLD_bwd_bnmk

Edit the Makefile to ensure compatibility with your system. Then, make the forward sweep executable.

make I\& tee make.bwd.log

Check that the executable ADJOINT_BWD is created.

\section{Benchmark the adjoint}

In this section, we will go through the steps listed in the introduction section about running the adjoint. The output files from each stage of the run could be compared with the ones we provide for benchmark.

\subsection{Run the forward model}

Running the forward sweep for the benchmark episode should be feasible after making modifications to the sample run script.

Change directory back to $\$$ ADJHOME/scripts.

Make a copy of the sample run script to modify it for your system.

cp run.adj.fwd.bnmk run.adj.fwd.bnmk. [system-specific-name]

Find all the instances of CHANGE to edit the run.adj.fwd.bnmk.[system-specificname] to direct the model to the location of the unzipped CMAQ adjoint benchmark data.

Successful completion of the run will produce ctm log files for each date (20070610 - 20070613) and will be indicated by Normal Completion of program DRIVER_FWD in the last log file.

The intermediate values of CMAQ state variables are saved to the checkpointing files at each synchronization time step for each science process, if necessary. In the output directory, the following checkpointing files should be present for each day (\$WDATE):

- CHEM_CHK_\$WDATE

- VDIFF_CHK_\$WDATE

- HA_RHOJ_CHK_\$WDATE

- VA_RHOJ_CHK_\$WDATE

- AERO_CHK_\$WDATE

- CLD_CHK_\$WDATE.

Comparisons can be made to the reference results provided in the benchmark data set. 


\subsection{Create adjoint forcing}

The backward sweep requires an adjoint forcing file, which can be thought of as similar to the emissions input of the forward sweep. The forcing file must be an IOAPI-compatible netCDF file for the purposes of being read accurately. The layer and species being forced should be indicated in the backward run script through environment variables ADJNLAYS_FRC and ADJNSPC_FRC.

Python is an efficient scripting tool for creating an adjoint forcing file from the concentration files output by the forward sweep. If your system does not have Python installed, consider using the free Anaconda distribution, which will make package management easy. To use the provided script, ensure that you have the netCDF4 and numpy packages installed.

To create an adjoint forcing to test the backward sweep, please modify scripts/BnmkAdjForcCalc.py where CHANGE indicates the need. Then, execute it.

python BnmkAdjForcCalc.py

Four files entitled ADJ_FORCE.20070610, etc. should be added to your output directory.

\subsection{Run the backward model}

In the same way the forward sweep run script template was configured for your system, refine the backward sweep run template.

cp run.adj.bwd.bnmk run.adj.bwd.bnmk. [system-specific-name]

For the sample adjoint forcing script, set the following specifications:

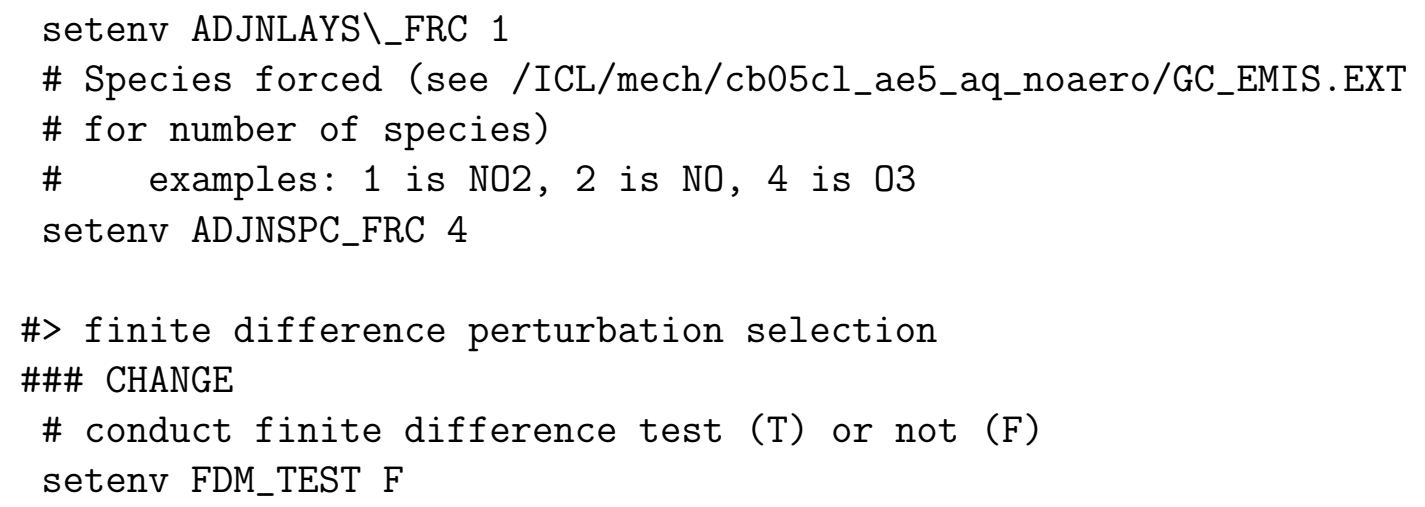

Execute the run script.

Comparisons can be made to the reference results provided in the benchmark data set.

\section{Finite difference test}

\subsection{The finite difference module}

A Fortran module (ADJ_FDM_TEST.F) comes with the forward model of the adjoint for finite difference test. The perturbation parameters such as perturbation species, types and locations can be specified via environment variables.

Below is an example in BASH. 


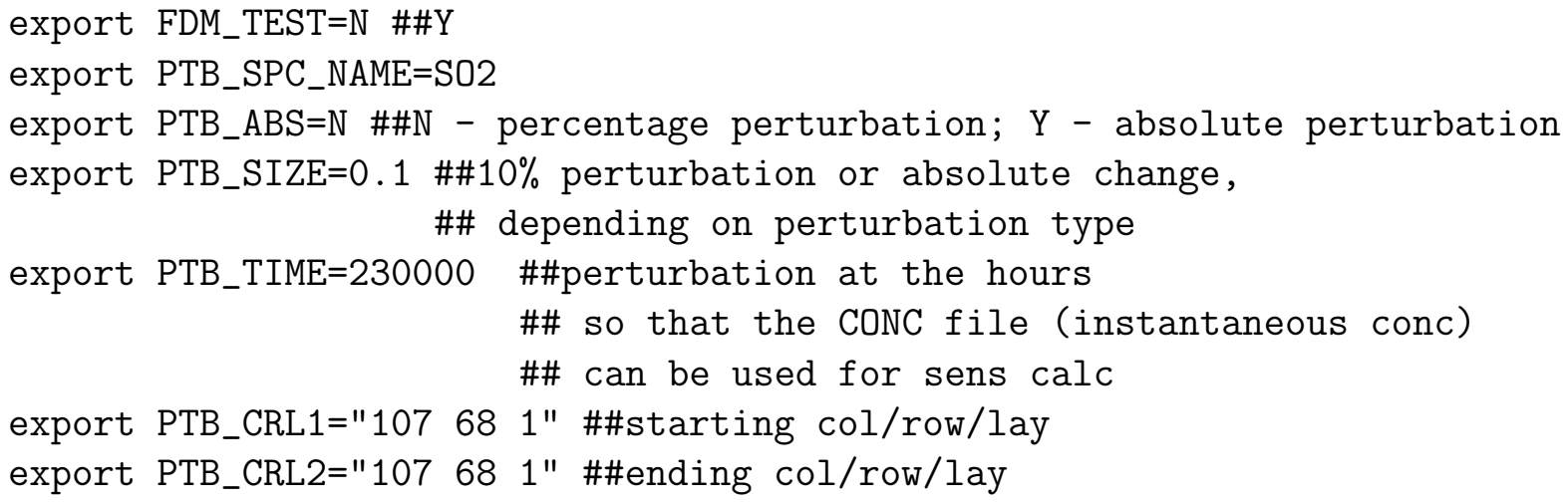

\subsection{Compare with the adjoint}

A perturbation introduced in the finite difference test would propagate over time across the computational domain and through other species. In other words, the finite difference comprises one perturbation source and multiple receptors. The finite difference test thus provides results that can be used to calculate the sensitivities of the affected apecies at all locations at all times to the perturbed species at the specic perturbation location at the specific perturbation time.

The adjoint, on the contrary, involes one receptor and multiple sources. One comparable sensitivity pair is generated from a single run of the models. In practice, a single receptor, which could be in the form of a function of several concentrations, might be used and the less computationally expensive finite difference test is then repeated to produce a desirable number of sensitivity pairs for comparison.

Another way is to reduce the full models to column/box models by turning off, for example, the transport and cloud mixing processes. This can be done by commenting out the corresponding science processes in sciproc.F. It is important that the science processes for the forward/backward models of the adjoint, and the finite difference test have to be consistent.

\section{Strategies to implement the adjoint}

\subsection{About checkpointing}

To generate the checkpointing files, a fixed synchronization time step is required. This can be achieved by assigning the same value to the environment variables, CTM_MAXSYNC and CTM_MINSYNC.

It is recommended to perform a test forward run with the default values of CTM_MAXSYNC (720 seconds) and CTM_MINSYNC (60 seconds) and then adopt the minimum value of the synchronization time step present in the log files for checkpointing files.

The checkpointing files could take gigantic storage space for long-period simulations with large domains and fine resolutions. To reduce the storage requirement, one might split the entire modeling period into smaller intervals, and only start generating for the last interal the checkpointing files which are required for the following backward model runs. The checkpointing files are then regenerated when needed for the other intervals. The environment variable, CREATE_CHK, can be used to switch on/off checkpointing generation. 


\subsection{About forcing}

Subroutine RD_FORCE_FILE in ADJOINT_FILES.F reads the forcing files and then applies the forcing to the corresponding species in your customized cost function.

Below is the piece of code that performs the forcing application. The names and indices of the species to be forced can be referred to Table 1. A DO loop, as demonstrated in the commented-out lines, can be used if multiple species are involved.

DO $\mathrm{R}=1$, LENROW

DO $\mathrm{C}=1$, LENCOL

!slz-pm25

! slz-pm25

!slz-pm25

!slz-pm25

!slz-pm25

$\&$

$\operatorname{ARRAY}(\mathrm{C}, \mathrm{R}, 1,4)=\operatorname{ARRAY}(\mathrm{C}, \mathrm{R}, 1,4)$ !slz layer $1 ; \mathrm{spc} 44$, o3

$+\operatorname{BUFFER}(\mathrm{C}, \mathrm{R}, 1) * \mathrm{FRCFAC}$

$\operatorname{ARRAY}(\mathrm{C}, \mathrm{R}, 1,1)=\operatorname{ARRAY}(\mathrm{C}, \mathrm{R}, 1,1)$ ! slz layer 1; spc\#1, no2

$+\operatorname{BUFFER}(\mathrm{C}, \mathrm{R}, 1) * \mathrm{FRCFAC}$

END DO

END DO 


\begin{tabular}{|c|c|c|c|c|c|c|c|}
\hline Index & Name & Index & Name & Index & Name & Index & Name \\
\hline 1 & NO2 & 36 & ETH & 71 & SESQ & 106 & NUMCOR \\
\hline 2 & $\mathrm{NO}$ & 37 & IOLE & 72 & SESQRXN & 107 & SRFATKN \\
\hline 3 & $\mathrm{O}$ & 38 & TOL & 73 & RHOJ & 108 & SRFACC \\
\hline 4 & O3 & 39 & CRES & 74 & ASO4J & 109 & SRFCOR \\
\hline 5 & NO3 & 40 & TO2 & 75 & ASO4I & 110 & AH2OJ \\
\hline 6 & O1D & 41 & TOLRO2 & 76 & ANH4J & 111 & AH2OI \\
\hline 7 & $\mathrm{OH}$ & 42 & OPEN & 77 & ANH4I & 112 & ANAJ \\
\hline 8 & $\mathrm{HO} 2$ & 43 & $\mathrm{CRO}$ & 78 & ANO3J & 113 & ANAI \\
\hline 9 & $\mathrm{~N} 2 \mathrm{O} 5$ & 44 & MGLY & 79 & ANO3I & 114 & ACLJ \\
\hline 10 & HNO3 & 45 & XYL & 80 & AALKJ & 115 & ACLI \\
\hline 11 & HONO & 46 & XYLRO2 & 81 & AXYL1J & 116 & ANAK \\
\hline 12 & PNA & 47 & ISOP & 82 & AXYL2J & 117 & ACLK \\
\hline 13 & $\mathrm{H} 2 \mathrm{O} 2$ & 48 & ISPD & 83 & AXYL3J & 118 & ASO4K \\
\hline 14 & $\mathrm{XO} 2$ & 49 & ISOPRXN & 84 & ATOL1J & 119 & ANH4K \\
\hline 15 & $\mathrm{XO} 2 \mathrm{~N}$ & 50 & TERP & 85 & ATOL2J & 120 & ANO3K \\
\hline 16 & NTR & 51 & TRPRXN & 86 & ATOL3J & 121 & $\mathrm{AH} 2 \mathrm{OK}$ \\
\hline 17 & $\mathrm{ROOH}$ & 52 & $\mathrm{SO} 2$ & 87 & ABNZ1J & 122 & AISO3J \\
\hline 18 & FORM & 53 & SULF & 88 & ABNZ2J & 123 & AOLGAJ \\
\hline 19 & ALD2 & 54 & SULRXN & 89 & ABNZ3J & 124 & AOLGBJ \\
\hline 20 & ALDX & 55 & ETOH & 90 & ATRP1J & 125 & NH3 \\
\hline 21 & PAR & 56 & ETHA & 91 & ATRP2J & 126 & SV_ALK \\
\hline 22 & $\mathrm{CO}$ & 57 & CL2 & 92 & AISO1J & 127 & SV_XYL1 \\
\hline 23 & MEO2 & 58 & $\mathrm{CL}$ & 93 & AISO2J & 128 & SV_XYL2 \\
\hline 24 & MEPX & 59 & HOCL & 94 & ASQTJ & 129 & SV_TOL1 \\
\hline 25 & $\mathrm{MEOH}$ & 60 & CLO & 95 & AORGCJ & 130 & SV_TOL2 \\
\hline 26 & $\mathrm{HCO} 3$ & 61 & FMCL & 96 & AORGPAJ & 131 & SV_BNZ1 \\
\hline 27 & FACD & 62 & $\mathrm{HCL}$ & 97 & AORGPAI & 132 & SV_BNZ2 \\
\hline 28 & $\mathrm{C} 2 \mathrm{O} 3$ & 63 & TOLNRXN & 98 & AECJ & 133 & SV_TRP1 \\
\hline 29 & PAN & 64 & TOLHRXN & 99 & AECI & 134 & SV_TRP2 \\
\hline 30 & PACD & 65 & XYLNRXN & 100 & A25J & 135 & SV_ISO1 \\
\hline 31 & $\mathrm{AACD}$ & 66 & XYLHRXN & 101 & A25I & 136 & SV_ISO2 \\
\hline 32 & $\mathrm{CXO} 3$ & 67 & BENZENE & 102 & ACORS & 137 & SV_SQT \\
\hline 33 & PANX & 68 & BENZRO2 & 103 & ASOIL & & \\
\hline 34 & ROR & 69 & BNZNRXN & 104 & NUMATKN & & \\
\hline 35 & OLE & 70 & BNZHRXN & 105 & NUMACC & & \\
\hline
\end{tabular}

Table 1: List of CGRID species of the cb05cl_ae5_aq mechanism. 


\subsection{About adjoint sensitivities}

The adjoint model produces two sensitivity files for each simulation day, i.e, the 'lgrid' file for sensitivities to concentrations and the 'lgrid_em' file for sensitivities to emissions.

The frequency of writing sensitivities to file can be controlled by the environment variable of ADJ_LGRID_FREQ. The value of 'OUTPUT_STEP' indicates that writing occurs at each output time step (hourly by default), which is defined by the environment variable of CTM_TSTEP; the value of 'SYNC_STEP' suggests writing at each synchronization time step. The latter produces times larger sensitivity files than the former and should be used with caution, especially for long-term simulations with large domain and fine resolution. 


\section{References}

[CMASWIKI, 2015] CMASWIKI (2015). Cmaq version 5.0 (february 2010 release) ogd - cmaswiki,. https://www . airqualitymodeling.org/index.php?title=CMAQ_ version_5.0_(February_2010_release)_OGD\&oldid=682. [Online; accessed 26September-2019].

[Shunliu Zhao, 2019] Shunliu Zhao, Amir Hakami, S. L. N. J. O. B. K. M. F. S. L. C. M. D. T. D. K. H. P. B. P. J. R. A. G. R. A. N. J. B. G. R. C. C. O. S. A. S. T. C. (2019). A multiphase cmaq version 5.0 adjoint. Geoscientific Model Development Discussions, 2019:1-37. doi:10.5194/gmd-2019-287. 